\title{
Structural and Magnetic Ordering in Fe-Ga Thin Films Examined by Mössbauer Spectrometry
}

\author{
T. Szumiata ${ }^{a}$, K. BrzózkA ${ }^{a}$, M. Gawroński $^{a}$, B. GórkA ${ }^{a}$, A. Javed $^{b}$, N.A. Morley ${ }^{b}$ \\ AND M.R.J. GIBBS ${ }^{b}$ \\ ${ }^{a}$ Department of Physics, Technical University of Radom, Krasickiego 54, 26-600 Radom, Poland \\ ${ }^{b}$ Department of Engineering Materials, University of Sheffield, Sheffield, S1 3JD, UK
}

\begin{abstract}
The detailed analysis of atomic and magnetic structure has been performed by Mössbauer spectroscopy for polycrystalline $\mathrm{Fe}_{80} \mathrm{Ga}_{20}$ films of different thickness ranging from $20 \mathrm{~nm}$ to $200 \mathrm{~nm}$. The films were deposited on $\mathrm{Si}(100)$ substrate using dual sputtering and evaporation method. Obtained CEMS spectra have been fitted with several Gaussian distributions of hyperfine magnetic field. The results point to the domination of BCC disordered $\mathrm{A} 2$ phase and the absence of $\mathrm{DO}_{3}$ and $\mathrm{L}_{2}$ phases in the films. Both hyperfine parameters and lattice constant reveal anomaly for $40 \mathrm{~nm}$ thick sample - presumably due to the change of the internal stress. Spin texture evolves from in-plane to out-of-the-plane configuration with increasing thickness.
\end{abstract}

PACS: 75.50.Bb, 75.70.Ak, 76.80.+y

\section{Introduction}

Fe-Ga alloy is characterized by low saturation field, low coercivity and high value of saturation magnetostriction constant. This material is applied in magnetic microelectromechanical systems (MagMEMS) [1]. Recently [2], polycrystalline $\mathrm{Fe}_{100-x} \mathrm{Ga}_{x}$ films $(14 \leq x \leq 32)$ deposited on $\mathrm{Si}(100)$ with a co-sputtering and evaporation technique were investigated. X-ray diffraction outcomes showed that all films had $\langle 110\rangle$ crystallographic texture normal to the film plane. The lattice parameter was an increasing function of gallium content up to $x=22$. Conversion Electron Mössbauer Spectroscopy (CEMS) results pointed to the predominance of the disordered A2 phase in all films. Also magnetoelastic measurements confirmed a suppression of $\mathrm{DO}_{3}$ ordered phase formation in the investigated films of various gallium atomic percentage. It provides a high magnetostriction value what could be very promising for the application in microelectromechanical systems. Main goal of present work was to investigate structural and magnetic ordering in Fe-Ga films of the constant Ga content but various thickness.

\section{Experimental details}

$\mathrm{Fe}_{80} \mathrm{Ga}_{20}$ films of different thickness, $t(20 \mathrm{~nm} \leq t \leq$ $200 \mathrm{~nm}$ ) were grown on $\mathrm{Si}(100)$ substrates (with the native oxide present) using a co-sputtering and evaporation technique [3]. The composition $\mathrm{Fe}_{80} \mathrm{Ga}_{20}$ has been chosen for this study as first peak in magnetostriction constant occurs around this composition in bulk single crystal [4] due to maxima in the magnetoelastic coupling constant $b_{1}[5]$. Also the magnetocrystalline anisotropy constants $K_{1}$ and $K_{2}$ are approximately zero at 20 at. $\% \mathrm{Ga}$ [6]. For the growth of $\mathrm{Fe}_{80} \mathrm{Ga}_{20}$ films, an Fe $(99.99 \%)$ target was DC sputtered with Fe power density $\left(P_{\mathrm{Fe}}\right) 1.48 \times 10^{4} \mathrm{Wm}^{-2}$ and Ga $(99.99 \%)$ was simultaneously thermally evaporated at a fixed relative rate $R_{\mathrm{Ga}}=0.3$ [a.u]. All films were grown at $p_{\mathrm{Ar}}=4 \mu \mathrm{bar}$ pressure. The composition of each film was measured using energy dispersive X-ray spectroscopy (EDX). Microstructural properties of the films were studied using $\mathrm{X}$-ray diffraction (XRD), atomic force microscope (AFM) and scanning electron microscopy (SEM) - to be published elsewhere. Conversion Electron Mössbauer Spectroscopy (CEMS) was used for the examination of structural order. ${ }^{57} \mathrm{Fe}$ CEMS spectra were collected at room temperature using a ${ }^{57} \mathrm{Co}: \mathrm{Rh}$ source. A gas flow type conversion electron detector in $2 \pi$ back scattering geometry (Model MM/CED-3) was used to count the resonant conversion 7.3 and $5.6 \mathrm{keV}$ electrons. The source was fixed to a vibrator operating in constant acceleration mode.

\section{Results and Discussion}

XRD data for all samples point to the $\left\langle{ }^{\prime} 110\right\rangle$ crystallographic texture perpendicular to the film. X-ray diffraction pattern presented in Fig. 1a does not containe any traces of $\mathrm{DO}_{3}$ superlattice reflections. The lattice parameter $a_{0}$ was determined from the $\langle 110\rangle$ peak centroid. The thickness film dependence of $a_{0}$ value (Fig. 1b) reveals nonmonotonic character. For the film thickness equal $40 \mathrm{~nm}$ lattice parameter seems to be minimal. A possible explanation of this fact could be the evolution 
of internal stress in course of growing process. We will come back to this question correlating XRD outcomes with CEMS results.
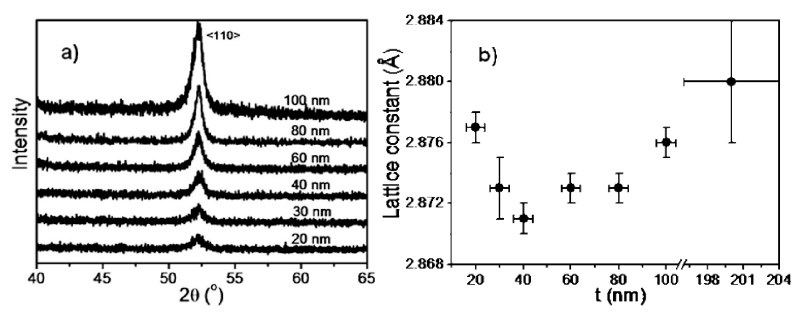

Fig. 1. (a) XRD patterns and (b) lattice parameter thickness dependence for $\mathrm{Fe}_{80} \mathrm{Ga}_{20}$ films.

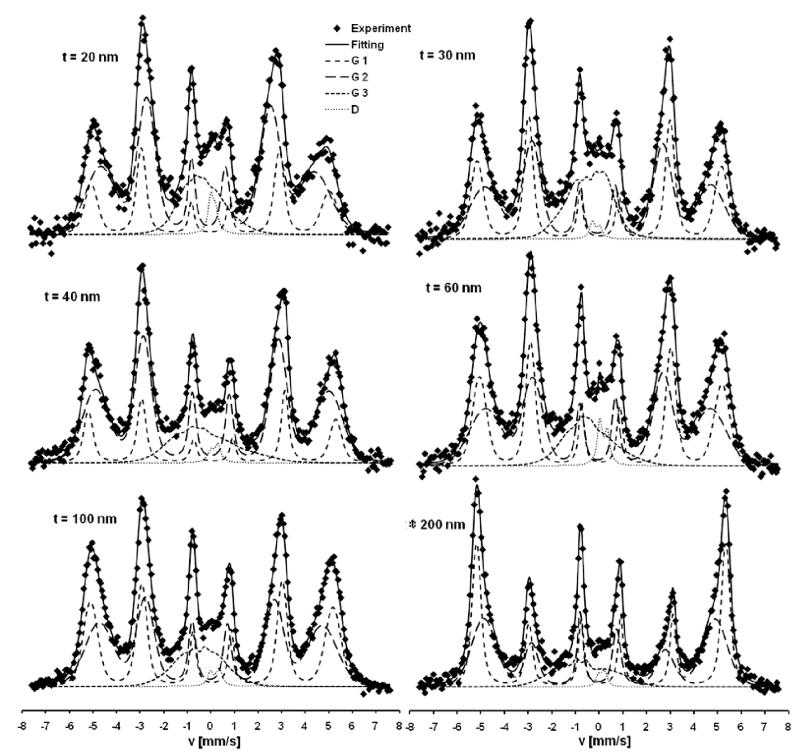

Fig. 2. CEMS spectra for $\mathrm{Fe}_{80} \mathrm{Ga}_{20}$ thin films of different thickness.

CEMS spectra (Fig. 2) have been fitted with Voigttype functions (Zeeman sextets convoluted with Gaussian hyperfine field distributions), what is a common procedure for disordered alloys [7, 8]. In order to minimize $\chi^{2}$ function combined gradient-genetic algorithm was utilized (like in previous paper [2]) transcribed for MS Excel VBA language. Experimental spectra with theoretical fits and corresponding hyperfine field distributions for $\mathrm{Fe}_{80} \mathrm{Ga}_{20}$ films of different thickness are presented in Fig. 2 and Fig. 3, respectively. The main component (G1) in all spectra corresponds to a narrow high-field distribution at the highest mean field value. It could be attributed to the $\mathrm{Fe}$ atoms with $8 \mathrm{Fe}$ the nearest neighbors in A2 (BCC) Fe-Ga phase. The second significant component (G2) comes from a hyperfine distribution of mean field values about $3 \mathrm{~T}$ lower than G1, and of higher standard deviation. It could be attributed also to the disordered BCC (A2-type) Fe-Ga solid solution, and corresponds to the replacement of $\mathrm{Fe}$ atoms by $\mathrm{Ga}$ atoms in the first and second neighbor shell of the resonant $\mathrm{Fe}$ atom. Actually, superposition of G1 and G2 symmetrical hyperfine field distributions could be regarded as one asymmetric distribution, which was reproduced in papers $[9,10]$ by multicomponent fitting within various models of the ordering in Fe-Ga alloys. Our work seems to be the first one concerning ultrathin layers and, consequently, utilizing not absorption spectrometry but CEMS technique. Due to the long time of measurements and not perfect statistics of spectra it was necessary to fit as simple model as possible (i.e. two main Gauss distributions). In the analyzed spectra no components characteristic for $\mathrm{DO}_{3}$ and $\mathrm{L}_{2}$ structure have been observed (in contrary to the reports [7, 10-11] for bulk and as quenched alloys as well as thick sputtered films). Central low field Gaussian subspectrum (G3) provides satisfactory fitting quality and supposedly describes Ga-rich phase of weak magnetism. Additional doublet (D) describes entirely nonmagnetic regions (probably at the uncovered surface).

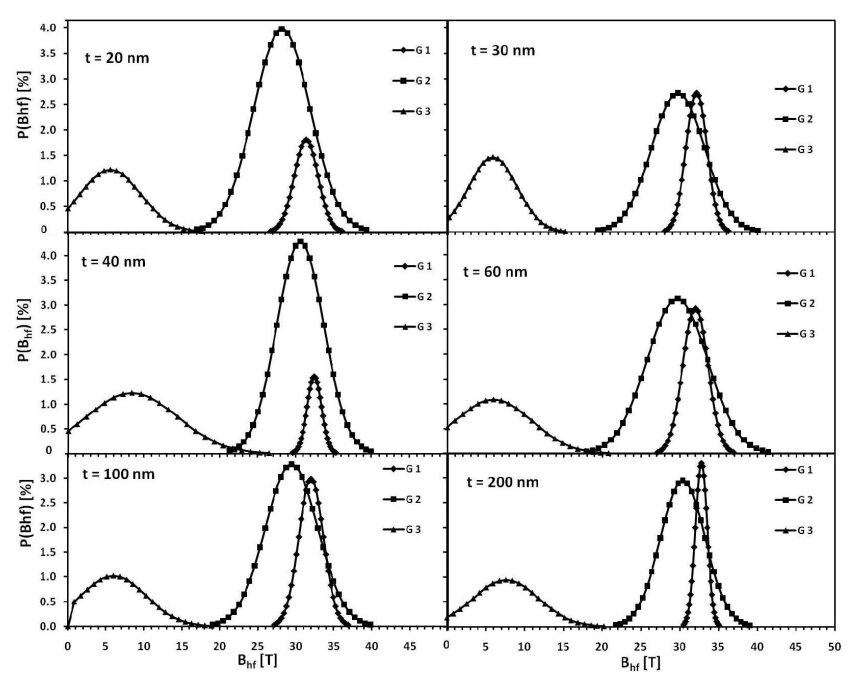

Fig. 3. Hyperfine distributions for $\mathrm{Fe}_{80} \mathrm{Ga}_{20}$ thin films of different thickness.

As shown in Figs. 4a-b mean hyperfine field and isomer shift reveal a maximum for the film $40 \mathrm{~nm}$ thick. As it was mentioned, this fact well coincides with the similar anomaly of lattice parameter. We tend to interpret this fact in terms of the change of the internal stress and growth process for the film of this definite thickness. We rather exclude the artifact connected with not constant gallium content, because EDX results confirmed a precise chemical content control after fabrication. However the question is still open and demands further investigation. By us it would be advisable to prepare ribbons of the same Ga content, apply a tensile stress and make measurements with conventional Mössbauer spectrometer.

It is worth adding that when thickness of films increases a contribution of weak magnetic components diminishes (Fig. 4c) what points to the better homogeneity of the samples. Another interesting feature of the stud- 

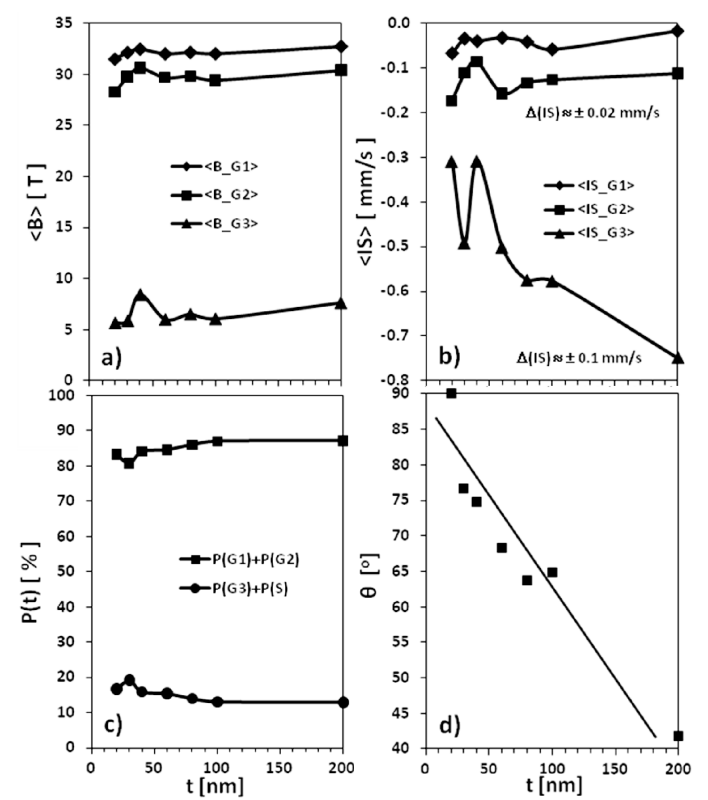

Fig. 4. (a) Mean hyperfine fields, (b) isomer shift (relatively to Fe foil), (c) components contributions and (d) magnetization direction vs. $\mathrm{Fe}_{80} \mathrm{Ga}_{20}$ films thickness.

ied system is a pronounced change of spin texture in the investigated films (Fig. 4d). The magnetization direction varies from in-plane to out-of-the-plane configuration when increasing thickness due to the evolution of perpendicular anisotropy $(\theta$ angle describes a direction of X-ray beam with respect to the magnetization vector). Future MOKE (Magnetooptical Kerr Effect) measurements could help with interpretation if the observed effect is a consequence of surface anisotropy or of a bulk-like anisotropy induced by the stress (internal or from the substrate) due to the high magnetostriction of the material.

\section{Conclusions}

CEMS studies confirmed that investigated films have disordered A2 structure and no traces of $\mathrm{DO}_{3}$ and $\mathrm{L}_{2}$ phases are observed, what is entirely consistent with XRD findings. A simple model with two main Gaussian distribution of hyperfine field seems to be sufficient for fitting and interpreting CEMS spectra. Correlation between hyperfine parameters and lattice parameter anomaly for $40 \mathrm{~nm}$ thick sample points to the important role of internal stress evolution during growth process. Spin texture reveals a transition from the in-plane to out-of-the-plane configuration with increasing thickness as a possible consequence of perpendicular magnetic anisotropy evolution in stressed high magnetostrictive material.

\section{References}

[1] M.R.J. Gibbs, IEEE Trans. Magn. 43, 2666 (2007).

[2] A. Javed, T. Szumiata, N.A. Morley, M.R.J. Gibbs, Acta Mater. 58, 4003 (2010).

[3] N.A. Morley, S.-L. Yeh, S. Rigby, A. Javed, M.R.J. Gibbs, J. Vac. Sci. Technol. A 26, 581, (2008).

[4] E.M. Summers, T.A. Lograsso, M. Wun-Fogle, J. Mater. Sci. 42, 9582 (2007).

[5] A.E. Clark, K.B. Hathaway, M. Wun-Fogle, J.B. Restorff, T.A. Lograsso, V.M. Keppens, G. Petculescu, R.A. Taylor, J. Appl. Phys. 93, 8621 (2003).

[6] S. Rafique, J.R. Cullen, M. Wuttig, J. Cui, J. Appl. Phys. 95, 6939 (2004).

[7] R.A. Dunlap, J.D. McGraw S.P. Farrell, J. Magn. Magn. Mater. 305, 315 (2006).

[8] K. Lagarec, D.G. Rancourt, Nucl. Instrum. Methods Phys. Res., Sect. B 129, 266 (1997).

[9] A. Blachowski, K. Ruebenbauer, J. Żukrowski, J. Przewoźnik, J. Alloys Compd. 455, 47 (2008).

[10] J.M. Borrego, J.S. Blazquez, C.F. Conde, A. Conde, S. Roth, Intermetallics 15, 193 (2007).

[11] R.A. Dunlap, N.C. Deschamps, R.E. Mar, S.P. Farrell, J. Phys. Condens. Matter 18, 4907 (2006). 